\title{
A SIMPLIFIED METHOD TO DESIGN FIXED-BED ADSORBERS FOR THE FREUNDLICH ISOTHERM
}

\author{
KenJI HASHIMOTO AND KouICHI MIURA* \\ Department of Chemical Engineering, Kyoto University, \\ Kyoto, 606
}

\begin{abstract}
An improved and simplified method to design fixed-bed adsorbers is developed under the assumption of a constant pattern of concentration distribution for the Freundlich isotherm systems. The intraparticle mass transfer rate is approximated by a modified linear-driving-force expression. The diagrams showing three parameters, which are necessary to estimate the break time, are presented and the procedures for predicting the break time and the breakthrough curve are presented.
\end{abstract}

\section{Introduction}

Basic equations for designing fixed-bed adsorption columns consist of a set of partial differential equations describing a bulk phase material balance of the adsorbate in the bed and the intraparticle mass transfer rate. These equations are generally complicated, therefore assumptions have been introduced to simplify the calculation. In relatively deep beds, the assumption of a constant pattern of concentration distribution approximately holds for favorable adsorption isotherms. The differential equation expressing the intraparticle mass transfer is often approximated by the linear-driving-force relation of Glueckauf ${ }^{3}$. These two approximations greatly reduce the amount of computation.

Simplified design methods based on the two approximations have been presented ${ }^{2,4,6}$. Most of them are concerned with the Langmuir type of isotherms, and are not with Freundlich isotherms which are frequently encountered in liquid phase adsorption processes.

In this paper an improved and simplified design method for the Freundlich isotherm is proposed. The procedures for predicting the break time and the breakthrough curve are presented.

\section{Development of an Approximate Design Method}

The velocity with which the adsorption front travels through the column, $v_{a}$, is formulated from the mass balance equation in the bed as follows ${ }^{8)}$ :

$$
v_{a}=\frac{u}{\varepsilon_{b}+\rho_{b}(\partial \bar{q} / \partial \bar{C})_{z}}
$$

where $u$ is the fluid superficial velocity, $\bar{q}$ is the mean solid phase concentration and $\bar{C}$ is the fluid phase Received April 9, 1976. concentration in the void space of the bed. Under the assumption of a constant pattern, $v_{a}$ remains constant regardless of the column length. Then the following well known equation is derived ${ }^{5,8)}$.

$$
\bar{q} / q_{0}=\bar{C} / C_{0}
$$

The Freundlich type of adsorption isotherm is written as follows:

$$
q / q_{0}=\left(C / C_{0}\right)^{\beta}
$$

The mass transfer rate in the fluid film surrounding particles is represented by

$$
\rho_{b} \frac{\partial \bar{q}}{\partial t}=k_{f} a_{v}\left(\bar{C}-C_{i}\right)
$$

where $C_{i}$ is the fluid phase concentration at the particle surface. With the linear-driving-force (L.D.F.) approximation, the intraparticle diffusion rate may be represented as follows:

$$
\rho_{b} \frac{\partial \bar{q}}{\partial t}=\eta k_{s} a_{v}\left(q_{i}-\bar{q}\right)
$$

where $q_{i}$ is the solid phase concentration in equilibrium with $C_{i}, \eta$ is a correction factor to improve the L. D. F. approximation, and $k_{s} a_{v}$ is related to the effective surface diffusivity, $D_{s}$, through the equation derived by Glueckauf ${ }^{3)}$.

$$
k_{s} a_{v}=\frac{15 D_{s}}{R^{2}} \rho_{b}
$$

The mass transfer rate is also expressed in terms of an overall volumetric coefficient, $K_{f} a_{v}$.

$$
\rho_{b} \frac{\partial \bar{q}}{\partial t}=K_{f} a_{v}\left(\bar{C}-C^{*}\right)
$$

where $C^{*}$ is in equilibrium with $\bar{q}$.

Eq. (4) can be integrated with the boundary condition, $\bar{x}=x_{B}$ at $t=t_{B}$, where $\bar{x}=\bar{C} / C_{0}$ and $x_{B}=$ $C_{B} / C_{0}$, to give the relation between $\bar{x}$ vs. $t$, that is the breakthrough curve, as follows: 


$$
t=t_{B}+\rho_{b} \frac{\gamma}{k_{f} a_{v}} \int_{x_{B}}^{\bar{x}} \frac{d \bar{x}}{\bar{x}-x_{i}}
$$

where $x_{i}=C_{i} / C_{0}$.

The time difference between the exhaustion and break points, $t_{E}-t_{B}$, can be obtained by integrating Eq. (7).

$$
t_{E}-t_{B}=\frac{\rho_{b} \gamma}{K_{f} a_{v}} \int_{x_{B}}^{x_{E}} \frac{d \bar{x}}{\bar{x}-x^{*}}
$$

where $\overline{K_{f} a_{v}}$ is the averaged $K_{f} a_{v}$ over the range between $x_{B}$ and $x_{E}$. The adsorption zone length, $z_{a}$, is represented by $v_{a}\left(t_{E}-t_{B}\right)$. Then by combining Eqs. (1) and (9), and by employing the fractional residual capacity of the adsorption zone ${ }^{7)}, f$, the following equation is finally obtained.

$$
t_{B}=\frac{\varepsilon_{b}}{u} z+\frac{\rho_{b} \gamma}{u}\left\{z-f_{K_{f} a_{v}}^{u} \int_{x_{B}}^{x_{E}} \frac{d \bar{x}}{\bar{x}-x^{*}}\right\}
$$

To estimate the break time on the basis of Eq. (10), three parameters, $\overline{K_{f} a_{v}}, \eta$ and $f$ must be obtained, because $\eta$ is necessary to determine $\overline{K_{f} a_{v}}$. Although for the Langmuir isotherm the correlations between $\overline{K_{f} a_{v}}$ and the ratio of $k_{f} a_{v}$ to $k_{s} a_{v} \gamma$ have been presented by Kawazoe et $a l^{6)}$ and Hiester et $a{ }^{6} .^{4}$, no relation has been published for the Freundlich isotherm. Usually $f$ is approximated to be 0.5 , however it is dependent on the shape of the breakthrough curve.

In the following sections, diagrams to evaluate the three parameters will be presented for the Freundlich isotherm.

\section{Diagram showing $\Psi$}

The time difference between the exhaustion and break points, $t_{E}-t_{B}$, can be also obtained by integrating Eq. (4)

$$
t_{E}-t_{B}=\frac{\rho_{b} \gamma}{k_{f} a_{v}} \int_{x_{B}}^{x_{E}} \frac{d \bar{x}}{\bar{x}-x_{i}}
$$

Combining Eqs. (9) and (11) yields

$$
\Psi=\frac{\overline{K_{f} a_{v}}}{k_{f} a_{v}}=\int_{x_{B}}^{x_{E}} \frac{d \bar{x}}{\bar{x}-x^{*}} / \int_{x_{B}}^{x_{E}} \frac{d \bar{x}}{\bar{x}-x_{i}}
$$

The numerator of Eq. (12) can be integrated analytically to give

$$
\int_{x_{B}}^{x_{E}} \frac{d \bar{x}}{\bar{x}-x^{*}}=\ln \frac{x_{E}}{x_{B}}+\frac{\beta}{1-\beta} \ln \frac{1-x_{B}^{(1-\beta) / \beta}}{1-x_{E}^{(1-\beta) / \beta}}
$$

To carry out the integration of the denominator of Eq. (12), the relation between $\bar{x}$ and $x_{i}$ must be obtained. By equating the right hand sides of Eqs. (4) and (5), and by use of Eqs. (2) and (3), the following equation is obtained,

$$
x_{i}^{\beta}=-\frac{\zeta}{\eta} x_{i}+\left(1+\frac{\zeta}{\eta}\right) \bar{x}
$$

where $\zeta=k_{f} a_{v} / k_{s} a_{v} \gamma$.

This equation was solved by the Regula-Falsi iteration method to obtain the relation between $\bar{x}$ and $x_{i}$. Thus the values of $\Psi$ are calculated as the function

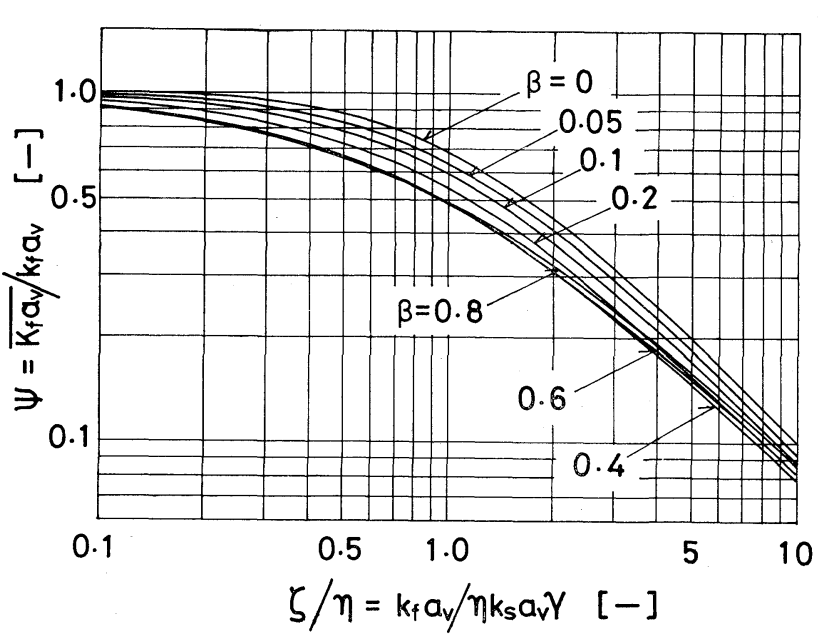

Fig. 1 Relation between $\Psi$ and $\zeta / \eta$

of $\zeta / \eta$ for several values of the Freundlich parameter $\beta$. The computed results are shown in Fig. 1, where $x_{B}$ and $x_{E}$ are taken as 0.1 and 0.9 , respectively.

\section{A correlation for factor $\eta$}

Factor $\eta$ was determined so that the length of the adsorption zone obtained by the proposed approximation method might agree with that computed by a numerical solution. This was carried out under the assumptions that a constant pattern was established and surface diffusion controlled the intraparticle mass transfer rate (see Appendix). The dimensionless time difference between the exhaustion and break points, which corresponds to the dimensionless adsorption zone length, was obtained as follows:

$$
\left(\theta_{E}-\theta_{B}\right)_{\mathrm{N} . \mathrm{S} .}=\left\{\frac{k_{f} a_{v}}{\rho_{b} \gamma}\left(t_{E}-t_{B}\right)\right\}_{\mathrm{N} . \mathrm{S} .}
$$

The following equation can be derived on the basis of the L. D. F. approximation

$$
\left\{\frac{k_{f} a_{v}}{\rho_{b} \gamma}\left(t_{E}-t_{B}\right)\right\}_{\text {L.D.F. }}=\int_{x_{B}}^{x_{E}} \frac{d \bar{x}}{\bar{x}-x_{i}}
$$

Substituting Eq. (12) into Eq. (16), and equating it with Eq. (15) yields

$$
\Psi=\int_{x_{B}}^{x_{E}} \frac{d \bar{x}}{\bar{x}-x^{*}} /\left(\theta_{E}-\theta_{B}\right)_{\mathrm{N} . \mathrm{s}} .
$$

The values of $\zeta / \eta$ corresponding to $\Psi$ is read from Fig. 1, and then the factor $\eta$ is evaluated. Fig. 2 shows the relation between $\eta$ and $\beta$ for several values of the parameter $\zeta$. $\eta$ increases almost linearly without depending on $\zeta$, and was found to be correlated by the equation.

$$
\eta=0.808+0.192 \beta
$$

The scattering of $\eta$ in the range of higher $\beta$ is probably attributed to the error in the numerical computation and the method for determining $\eta$.

Estimation of $\boldsymbol{f}$

The $f$ value, which is involved in the $t_{B}$ term (see Eq. (10)), can be determined from the following 


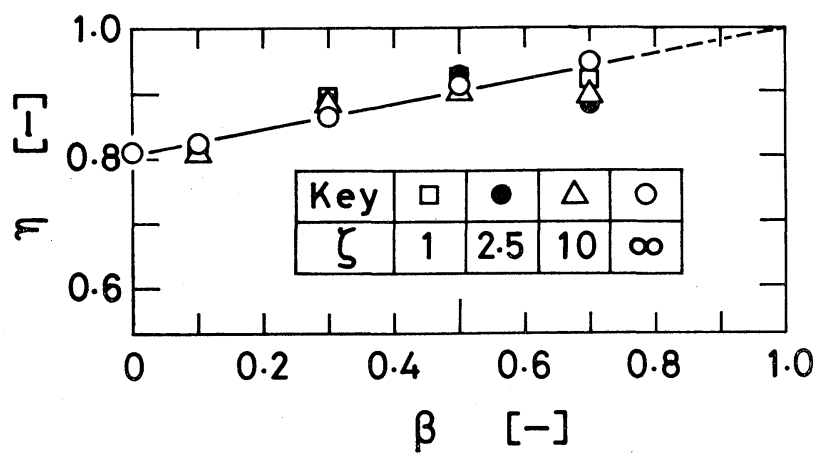

Fig. 2 Correction factor of solid volumetric coefficient $\eta$ vs. Freundlich coefficient $\boldsymbol{\beta}$

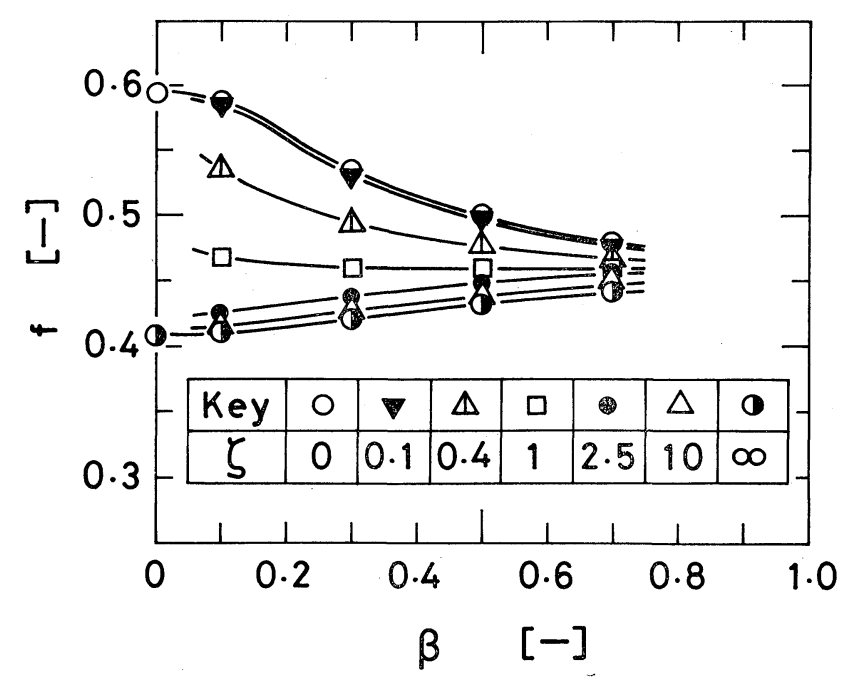

Fig. 3 Fractional residual capacity of the adsorption zone $f$ vs. $\beta$

overall mass balance in the bed:

$$
\left(\rho_{b} q_{0}+\varepsilon_{b} C_{0}\right) z=u C_{0} \int_{0}^{\infty}(1-\bar{x}) d t=u C_{0} \int_{0}^{1} t d \bar{x}
$$

To calculate the integrand in Eq. (19), Eq. (8) must be evaluated. Especially when either the fluid film or the solid phase mass transfer is controlling, Eq. (8) can be analytically integrated, and then the value of $f$ is easily evaluated. However, numerical integration must be performed in the cases where both resistances are significant. In Fig. 3 the $f$ values which were determined for $x_{B}=0.1$ are shown. Unlike $\eta$ the parameter $f$ depends considerably on $\zeta$. In the case where $\beta=0.5$ and the external mass transfer is controlling $(\zeta=0)$, it was found that $f$ is 0.5 and that the shape of the breakthrough curve is symmetrical about $\bar{x}=0.5$.

\section{Application of the Approximation Method \\ Prediction of $\boldsymbol{t}_{B}$}

If the adsorption isotherm and mass transfer coefficients are known, the break time, $t_{B}$, is easily estimated by the following procedure: (1) read $\eta$ from Fig. 2, (2) read $\Psi$ from Fig. 1 and calculate $\overline{K_{f} a_{v}}$, (3)

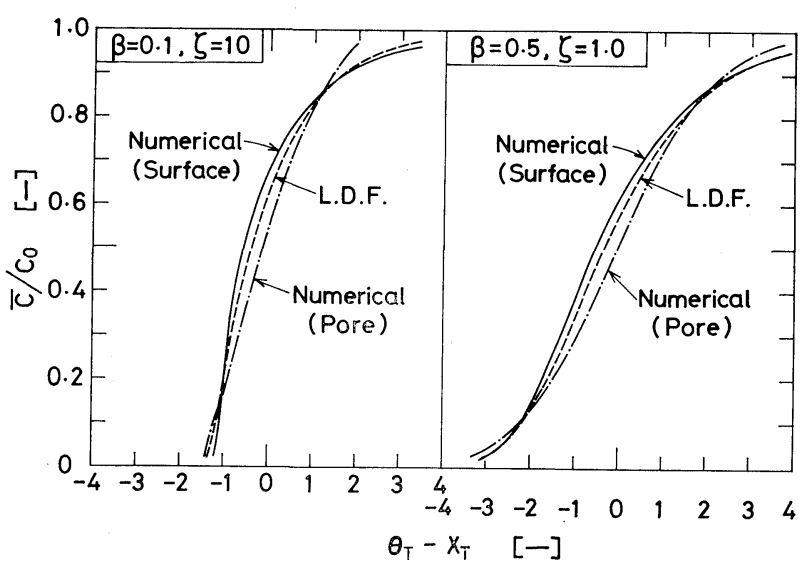

Fig. 4 Comparison between proposed approximation method (L. D. F.) and numerical solutions

obtain $f$ from Fig. 3, (4) calculate the integrand in Eq. (10) by using Eq. (13) and (5) substitute these values into Eq. (10) to yield $t_{B}$.

Estimation of a full breakthrough curve

The evaluation of the full breakthrough curve, which is a little more tedious, can be performed in the following manner: (1) estimate $t_{B}$ in the same manner as in the previous section, and (2) obtain $x_{i}$ for a number of $\bar{x}$ values using Eq. (14), and then integrate Eq. (8) to obtain the relation between $t$ and $\bar{x}$.

\section{Discussion}

Breakthrough curves (broken lines) calculated by the proposed simplified method for two cases, $\beta=0.1$, $\zeta=10.0$ and $\beta=0.5, \zeta=1.0$, are shown in Fig. 4 , and are compared with the corresponding numerical solutions (solid lines) based on surface diffusion kinetics. The abscissa is $\left(\theta_{T}-X_{T}\right)$ which corresponds to $N_{T}(T-1)$ used by Fleck et al. ${ }^{1}$.

The solid line and the broken line show fairly good agreement in each case. This fact suggests that the proposed method is valid when surface diffusion is dominant within the particle. If only the break time is necessary, which is the case in industrial applications, the L. D. F. approximation gives almost the same results that are obtained by the numerical solution.

The proposed method was developed as an approximation for the case of surface diffusion kinetics. However, it seems that the method is approximately applicable to the case where pore diffusion is controlling the intraparticle mass transfer. In this case the same breakthrough curve can be obtained if $k_{s} a_{v}$ is approximated by

$$
k_{s} a_{v}=\frac{15 D_{p}\left(1-\varepsilon_{b}\right)}{R^{2}} \cdot \frac{C_{0}}{q_{0}}
$$

where $D_{p}$ is the effective pore diffusivity. The applicability of the approximation method can be examined by comparing the obtained breakthrough 
curves (broken lines) with the numerical solutions (chain lines) based on pore diffusion kinetics as shown in Fig. 4. It can be concluded that this simplified method is applicable particularly when only the break time is required.

It is also possible to estimate the intraparticle diffusivities from the break and exhaustion times obtained experimentally, $\left(t_{B}\right)_{\exp }$ and $\left(t_{E}\right)_{\text {exp }}$. By use of Eqs. (11) and (12), $(\Psi)_{\exp }$ can be represented as follows:

$$
(\Psi)_{\mathrm{exp}}=\rho_{b} \gamma \int_{x_{B}}^{x_{E}} \frac{d \bar{x}}{\bar{x}-x^{*}} / k_{f} a_{v}\left\{\left(t_{E}\right)_{\mathrm{exp}}-\left(t_{B}\right)_{\mathrm{exp}}\right\}
$$

The numerator of Eq. (21) can be calculated using Eq. (13). Then the value of $\zeta / \eta$ is obtained against $(\Psi)_{\text {exp }}$ from Fig. 1, and the $\eta$ value is read from Fig. 2. Thus the value of $k_{s} a_{v}$ is evaluated, and $D_{s}$ is estimated by use of Eq. (6) as

$$
D_{s}=\frac{R^{2}}{15} \cdot \frac{k_{s} a_{v}}{\rho_{b}}
$$

If pore diffusion is considered to be dominant within the particle, the pore diffusivity, $D_{p}$, may be approximately calculated by

$$
D_{p}=\frac{R^{2}}{15} \cdot \frac{k_{s} a_{v}\left(q_{0} / C_{0}\right)}{\left(1-\varepsilon_{b}\right)}
$$

\section{Conclusion}

A simplified design method of fixed-bed adsorbers for the Freundlich isotherm was developed under the assumption of a constant pattern. Diagrams for evaluating the three unknown parameters in this method were presented. The break time and breakthrough curve were estimated by means of those diagrams, and were compared with the numerical solution based on surface diffusion kinetics.

The approximation method showed good agreement with the corresponding numerical solution. When only the break time was necessary, this method gave almost the same results as were predicted by the numerical solution based on pore diffusion kinetics.

\section{Appendix}

The basic equations for the case of surface diffusion kinetics are written in dimensionless forms as follows: Bed:

$$
\frac{\partial \bar{x}}{\partial \theta}=\bar{x}-x_{i}
$$

Intraparticle:

$$
15 \zeta \frac{\partial y}{\partial \theta}=\frac{\partial^{2} y}{\partial \xi^{2}}+\frac{2}{\xi} \frac{\partial y}{\partial \xi}
$$

Boundary condition:

$$
5 \zeta\left(\bar{x}-x_{i}\right)=\left(\frac{\partial y}{\partial \xi}\right)_{\xi=1}
$$

where $y=q / q_{0}$ and $\xi=r / R$.

The bed and intraparticle equations were replaced by the forward finite difference scheme and the Crank-Nicolson implicit scheme, respectively. Finally the following equations were obtained:

$$
\begin{aligned}
& \frac{\Delta \theta}{2} x_{M, n+1}+\left[1-\frac{\Delta \theta}{2}\right] \bar{x}_{n+1}=\bar{x}_{n}+\frac{\Delta \theta}{2}\left[\bar{x}_{n}-x_{M, n}\right] \\
& -\frac{\alpha}{15 \zeta} y_{M-1, n+1}+\left[1+\frac{\alpha}{15 \zeta}\right] y_{M, n+1}=y_{M, n}+\frac{\alpha}{15 \zeta}\left[y_{M-1, n}-y_{M, n}\right] \\
& \quad+\frac{\Delta \theta}{3}(M+1)\left[\bar{x}_{n+1}-x_{M, n+1}+\bar{x}_{n}-x_{M, n}\right] \\
& -\frac{m-1}{6 m} \frac{\alpha}{5 \zeta} y_{m-1, n+1}+\left[1+\frac{\alpha}{15 \zeta}\right] y_{m, n+1}-\frac{m+1}{6 m} \frac{\alpha}{5 \zeta} y_{m+1, n+1} \\
& \quad=y_{m, n}+\frac{1}{6 m} \frac{\alpha}{5 \zeta}\left\{(m-1) y_{m-1, n}-2 m y_{m, n}+(m+1) y_{m+1, n}\right\} \\
& {\left[1+\frac{\alpha}{15 \zeta}\right] y_{1, n+1}-\frac{\alpha}{15 \zeta} y_{2, n+1}=\left[1-\frac{\alpha}{15 \zeta}\right] y_{1, n}+\frac{\alpha}{15 \zeta} y_{2, n}}
\end{aligned}
$$

where $\alpha=\Delta_{\theta} / \Delta \xi^{2}, m$ is the space index within the particle, $n$ is the time index $(n=0,1,2, \ldots), \Delta \xi$ is the radial increment and $\Delta \theta$ is the time increment. The spherical particle was divided into 10 shells, and $x_{M, n}$ corresponds to $x_{i}$. An appropriate value of $\alpha$ was found to be 1.0 .

To start the numerical integration, the boundary condition was assumed to be $\bar{x}=10^{-3}$ at $\theta=\theta_{0}$. This means a small value $\left(=10^{-3}\right)$ was assigned to $\bar{x}_{0}$ at $\theta_{\mathrm{N} . \mathrm{s} .}=0$. The dimensionless time $\theta_{\text {N.s. }}$ was measured from $\theta_{0}$. The other values at the 0 th time level were assumed to be 0 .

At first $x_{M, 1}$ was replaced by $x_{M, 0}(=0)$, and Eq. (A-4) was solved to give $\bar{x}_{1}$. Then Eqs. (A-5) to (A-7) constitute the tridiagonal form of simultaneous linear equations of dimension $M$. These equations were solved by the Thomas method to obtain $y_{m, 1}(m=1,2, \cdots, M)$. By use of the Simpson rule the first approximate value of $\bar{y}_{1}\left(=\bar{x}_{1}\right)$ could be obtained. The new value of $x_{M, 1}$ was set equal to $\left(y_{M, 1}\right)^{1 / \beta}$, and Eqs. (A-4) to (A-7) were again solved to obtain the second approximate value of $\bar{x}_{1}$. This procedure was continued until $\bar{x}_{1}$ converged within $10^{-4}$. Usually, two iterations were required for the convergence.

By changing the index $n$, the values of $\bar{x}$ were successively

\begin{tabular}{|c|c|}
\hline$a_{v}$ & $=$ specific particle surface area in bed \\
\hline$C$ & $=$ fluid phase concentration \\
\hline $\bar{C}$ & $=$ concentration in the void space of bed $\left[\mathrm{mg} / \mathrm{cm}^{3}\right]$ \\
\hline$C^{*}$ & $=$ concentration in equilibrium with $\bar{q} \quad\left[\mathrm{mg} / \mathrm{cm}^{3}\right]$ \\
\hline$D_{p}$ & $=$ effective pore diffusivity \\
\hline$D_{s}$ & $=$ effective surface diffusivity $\quad\left[\mathrm{cm}^{2} / \mathrm{sec}\right]$ \\
\hline$f$ & $\begin{array}{l}=\text { fractional residual capacity of the adsorp- } \\
\text { tion zone }\end{array}$ \\
\hline$K_{f}$ & $=$ overall mass transfer coefficient $\quad[\mathrm{cm} / \mathrm{sec}]$ \\
\hline$\overline{K_{f} a_{v}}$ & $\begin{aligned}= & \text { averaged } K_{f} a_{v} \text { over the range between } \\
& x_{B} \text { and } x_{E}\end{aligned}$ \\
\hline$k_{f}$ & $=$ fluid film mass transfer coefficient $\quad[\mathrm{cm} / \mathrm{sec}]$ \\
\hline$k_{s}$ & $\begin{array}{l}=\text { coefficient concerning the intraparticle } \\
\text { mass transfer rate }\left[\mathrm{g} / \mathrm{cm}^{2} \cdot \mathrm{sec}\right]\end{array}$ \\
\hline$M$ & $=$ number of radial shells \\
\hline
\end{tabular}
computed for several $\beta$ and $\zeta$ values. However, the $\theta_{0}$ value must be determined to obtain the relation $\bar{x}$ vs. $\theta$. This was determined from the overall mass balance. Equation (19) can be rewritten in dimensionless form as

$$
\int_{0}^{1} \theta d \bar{x}=\int_{0}^{1}\left(\theta_{0}+\theta_{\mathrm{N} . \mathrm{s} .}\right) d \bar{x}=X
$$

The integrand of Eq. (A-8) can be estimated by graphical integration, and the $\theta_{0}$ value was determined. The general plots (Fig. 4) were obtained using the general dimensionless parameters, $\theta_{T}$ and $X_{T}$. A similar procedure was employed for the case of pore diffusion kinetics.

\section{Nomenclature}




$\begin{array}{ll}N_{T}(T-1)= & \text { dimensionless parameter of Fleck } \text { et } a l .^{1)} \\ q & =\text { solid phase concentration } \\ \bar{q} & =\text { averaged solid phase concentration } \\ R & =\text { particle radius } \\ t & =\text { time } \\ u & =\text { superficial fluid velocity } \\ v_{a} & \text { velocity with which the adsorption front } \\ & \text { travels through the column } \\ X & =\left(k_{f} a_{v} / u\right) z \\ X_{T} & =\text { dimensionless fluid phase concentration } \\ x & \left(=C / C_{0}\right) \\ x^{*} & =C^{*} / C_{0} \\ y & =q / q_{0} \\ z & =\text { column length } \\ z_{a} & \text { adsorption zone length } \\ \alpha & =\Delta \theta / \Delta \xi^{2} \\ \beta & = \\ \gamma & =q_{0} / C_{0} \\ \varepsilon_{b} & =\text { void fraction of the bed } \\ \zeta & =k_{f} a_{v} / k_{s} a_{v} \gamma \\ \theta & =\left(k_{f} a_{v} / \rho_{b} \gamma\right)\left\{t-\left(\varepsilon_{b} / u\right) z\right\} \\ \theta_{T} & =\left\{k_{f} a_{v} / \rho_{b} \gamma(1+\zeta)\right\}\left\{t-\left(\varepsilon_{b} / u\right) z\right\} \\ \xi & =r / R \\ \rho_{b} & =\text { bed density } \\ & \end{array}$

\begin{tabular}{|c|c|c|}
\hline $\begin{array}{r}{[-]} \\
{[\mathrm{mg} / \mathrm{g}]} \\
{[\mathrm{mg} / \mathrm{g}]}\end{array}$ & \multicolumn{2}{|c|}{$\langle$ Subscripts $\rangle$} \\
\hline$[\mathrm{cm}]$ & $B$ & $=$ break point \\
\hline [sec] & $E$ & $=$ exhaustion point \\
\hline$[\mathrm{cm} / \mathrm{sec}]$ & $\exp$ & $=$ experimental \\
\hline & $i$ & $=$ outer surface of the particle \\
\hline$[\mathrm{cm} / \mathrm{sec}]$ & L.D.F. & $=$ linear-driving-force \\
\hline$[-]$ & & $=$ radial index in the numerical solution \\
\hline$[-]$ & $\begin{array}{l}\text { N.S. } \\
n\end{array}$ & $\begin{array}{l}=\text { numerical solution } \\
=\text { time index in the numerical solution }\end{array}$ \\
\hline$[-]$ & 0 & $=$ column inlet \\
\hline$[-]$ & \multicolumn{2}{|c|}{ Literature Cited } \\
\hline $\begin{array}{l}{[\mathrm{cm}]} \\
{[\mathrm{cm}]}\end{array}$ & $\begin{array}{l}\text { 1) Fle } \\
\text { Fun } \\
\text { 2) Gar }\end{array}$ & $\begin{array}{l}\text { R. D., D. J. Kirwan and K. R. Hall: Ind. Eng. Chem., } \\
\text { nentals, 12, } 95 \text { (1973). } \\
\text { D. R. and D. M. Ruthven: AIChE J., 21, } 200 \text { (1975). }\end{array}$ \\
\hline$[-]$ & \multicolumn{2}{|c|}{ 3) Glueckauf, E.: Trans. Faraday Soc., 51, 1540 (1955). } \\
\hline $\begin{array}{r}{[-]} \\
{\left[\mathrm{cm}^{3} / \mathrm{g}\right]}\end{array}$ & \multicolumn{2}{|c|}{$\begin{array}{l}\text { 4) Hiester, N. K., S. B. Radding, R. L. Nelson and T. Ver- } \\
\text { mulen: AIChE J., 2, } 404 \text { (1956). }\end{array}$} \\
\hline$[-]$ & \multicolumn{2}{|c|}{$\begin{array}{l}\text { 5) Kawazoe, K.: "Shin Kagaku Kogaku Koza”, VI-3b, Nikkan } \\
\text { Kogyo Shinbunsha (1958). }\end{array}$} \\
\hline$[-]$ & \multicolumn{2}{|c|}{$\begin{array}{l}\text { 6) Kawazoe, K. and Y. Fukuda: Kagaku Kögaku, 29, } 374 \\
\text { (1965). }\end{array}$} \\
\hline$[-]$ & \multicolumn{2}{|c|}{ 7) Michaels, A. S.: Ind. Eng. Chem., 44, 1922 (1952). } \\
\hline$\left[\mathrm{g} / \mathrm{cm}^{3}\right]$ & \multicolumn{2}{|c|}{ 8) Wevers, C. J. H.: Chem. Eng. Sci., 10, 171 (1959). } \\
\hline
\end{tabular}

\title{
THE EFFECTIVENESS FACTOR OF A CATALYST PELLET IN THE LIQUID-PHASE HYDROGENATION OF STYRENE
}

\author{
KoEI KAWAKAMI*, ShigetSUgu URA ${ }^{1}$ \\ AND KOICHIRO KUSUNOKI \\ Department of Chemical Engineering, Kyushu University, \\ Fukuoka, 812
}

\begin{abstract}
The reaction characteristics of a catalyst pellet is examined in the liquid-phase hydrogenation of styrene in a stirred basket reactor. The catalyst used is a $0.5 \% \mathrm{Pt}-\mathrm{Al}_{2} \mathrm{O}_{3}$ cylindrical pellet. The intrinsic kinetic expression of the Langmuir-Hinshelwood form is fashioned from the rate measurements in a stirred slurry reactor in which the finely crushed pellets are suspended. With the catalyst pellets the pore diffusion of hydrogen is observed to be significant.

The effectiveness factor of the catalyst pellet is computed on the basis of a simplified theoretical model. The connection between the catalytic effectiveness factors obtained experimentally and theoretically makes it possible to estimate the effective diffusivity of hydrogen and the tortuosity factor of the catalyst pellet. The tortuosities ranging from 2.5 to 3.2 , secured at the three temperature levels, are shown to be reasonable values for the pore diffusion within a liquid filled catalyst.
\end{abstract}

\section{Introduction}

The phenomena of the intraparticle diffusion with adsorption or catalytic reaction within a liquid-filled

Received February 4, 1976.

Presented at the 9th Autumn Meeting of The Soc. of Chem. Engrs., Japan, October 17, 1975.

1 Idemitsu Kosan Co. Ltd., porous solid have been studied and reported by many workers ${ }^{1,2,5)}$. In these studies, a common interest has been focused on the magnitude of the tortuosity factor in the light of the published values ${ }^{9)}$ for gas-filled pores. There have been few references directly concerned with liquid-phase hydrogenation. The tortuosity factor of 3.9 was first found by Satterfield et al.$^{8}$, for the reduction of $\alpha$-methyl styrene on a $0.5 \% \mathrm{Pd}-\mathrm{Al}_{2} \mathrm{O}_{3}$ 